\title{
The Casimir Topological Effect and a Proposal for a Casimir-Dark Energy Nano Reactor*
}

\author{
Mohamed S. El Naschie \\ Department of Physics, University of Alexandria, Alexandria, Egypt \\ Email: Chaossf@aol.com
}

Received 2 March 2015; accepted 16 March 2015; published 18 March 2015

Copyright (C) 2015 by author and Scientific Research Publishing Inc.

This work is licensed under the Creative Commons Attribution International License (CC BY). http://creativecommons.org/licenses/by/4.0/

(c) (i) Open Access

\section{Abstract}

A basically topological interpretation of the Casimir effect is given as a natural intrinsic property of the geometrical topological structure of the quantum-Cantorian micro spacetime. This new interpretation compliments the earlier conventional interpretation as vacuum fluctuation or as a Schwinger source and links the Casimir energy to the so called missing dark energy density of the cosmos. We start with a general outline of the theoretical principle and basic design concepts of a proposed Casimir dark energy nano reactor. In a nutshell the theory and consequently the actual design depends crucially upon the equivalence between the dark energy density of the cosmos and the faint local Casimir effect produced by two sides boundary condition quantum waves. This Casimir effect is then colossally amplified as a one sided quantum wave pushing from the inside on the one sided Möbius-like boundary with nothing balancing it from the non-existent outside. In view of the present theory, this one sided Möbius-like boundary of the holographic boundary of the universe is essentially what leads to the observed accelerated expansion of the cosmos. Thus in principle we will restructure the local topology of space using material nanoscience technology to create an artificial local high dimensionality with a Dvoretzky theorem like volume measure concentration. Needless to say the entire design is based completely on the theory of quantum wave dark energy proposed by the present author. The quintessence of the present theory is easily explained as the $\phi^{3}$ intrinsic Casimir topological energy where $\phi=(\sqrt{5}-1) / 2$ produced from the zero set $\phi$ of the quantum particle when we extract the empty set quantum wave $\phi^{2}$ from it and find $\phi-\phi^{2}=\phi^{3}$ by restructuring space via plates similar to that of the classical Casimir experiments but with some modification.

\section{Keywords}

Casimir Effect, Dark Energy, E-Infinity, Cantorian Spacetime, Nano Reactor, Free Energy

*Dedicated to President Abdul Fattah Al-Sisi. 


\section{Introduction and Motivation}

It is difficult, if not near impossible to give even a glimpse into the past work done in a vibrant and vast field like the zero point vacuum energy which spans the entirety of fundamental theoretical and experimental physics. So rather than attempting the unfeasible, we have included in the present paper a very large amount of references and the reader is referred to these publications and the references therein [1]-[58].

The present paper has two different messages to communicate, a scientific one centred around the quantum vacuum as a source of energy [1]-[58] and a socio-economical, political message that we must invest in this new revolutionary source of energy [59]-[61]. The idea of zero point energy and the fluctuation of vacuum may seem at first glance to be more science fiction than science fact. However, there are, and since quite some time, a host of hard core experimental evidence that the vacuum may be more real ad fundamental than most of what we habitually consider the materialistic reality of physical phenomenon [1]-[58]. We just need to mention in this context the Lamb shift, Schwinger correction [62]-[66] and the van der Waals forces to realize how physical and real the vacuum is [22]-[24] [28]. None the less, and we do not think it is a minority opinion, nothing could be more impressive and inspiring as the Casimir effect [22]-[24]. This effect is a natural consequence and fundamental aspect of quantum field theory. There are at least two fundamental interpretations of this miraculous effect [63] [64]. The first is loosely connect to boundary conditions and the zero point quantum vacuum fluctuation which may be the common way of looking at the Casimir effect within the working physicists community. The second, which may be more theoretical and fundamental, is to see Casimir as a source in the mould of J. Schwinger's way of thinking and not far from the Casimir operators of quantum field theory [62] [63] [66]. Thus we could look upon the Casimir effect as a cousin of Hawking's negative energy fluctuation around a black hole or as Unruh's temperature for an accelerated, observed in a Rindler wedge, universe. Alternatively we could follow Schwinger's ideas and see it as something related to a fundamental mathematical scenario such as the Banach-Tarski theorem advanced for the first time in the cosmology of the big bang by the present author [67] [68].

In the present paper however we opted for a rather different point of viewing the Casimir effect as a natural topological necessity of a Cantorian spacetime fabric which was woven from an infinite number of zero Cantor sets and empty Cantor sets [37]. The zero set is taken following von Neumann-Connes dimensional function to model the quantum particles while the empty set models the quantum wave. Following this road we come we come to realize that the Casimir latent energy is nothing but the universal fluctuation $\phi^{3}$ which gives birth to the core of Cantorian-fractal spacetime by inversion $1 / \phi^{3}=4+\phi^{3}=4.23606797$ where $\phi=(\sqrt{5}-1) / 2$ [52] [53]. This is nothing but the difference between the Hausdorff dimension of the particle zero set $\phi$ and the wave empty set $\phi^{2}$. The result not surprisingly is almost equal to double the value found using imaginative modifycation of the classical Casimir experiment by Zee [62] who found the dimensionless Casimir energy to be $\pi / 24 \simeq 0.1308$ [62]. Using E-infinity methodological reasoning, the exact value of Zee in the limit must be the ratio of the dimensionality of a Calabi-Yau transfinite manifold $6+k=6.18033889$ and the transfinite dimension of bosonic string theory, i.e. 26.18033989. That means $(6+k) /(26+k)=\phi^{3} / 2$. Needless to say, the division by 2 is due to the subdivision of the “vacuum” of $E$-infinity theory and is analogous to dividing Hardy's entanglement $P(H)=\phi^{5}$ by 2 to obtain the density of the ordinary measurable energy of the cosmos

$E(O)=\left(\phi^{5} / 2\right) m c^{2}$. The dimensional quantity analogous to $m c^{2}$ for the Casimir effect is trivially clear to be $\hbar c$ where $c$ is the speed of light and $\hbar$ is the Planck quantum. From this new topological interpretation it becomes obvious that Casimir $\phi^{3}$ is the counterfactual or global part of Hardy's entanglement $P(H)=\left(\phi^{3}\right)\left(\phi^{n}\right)$ where $\mathrm{n}$ is the number of quantum particles and is found for $n=0$. It is therefore closely related to the Unruh temperature where $n=1$, the Immirzi parameter $n=3$ as well as Hardy's generic quantum-topological entanglement $n=2$. These insights are not only simple mathematical insights. It goes far beyond that and suggests that Casimir energy and dark energy are two sides of the same coin, differing only with regard to exo and endo boundary conditions [6] [7] which will be made clearer in the main body of the present work. Second, by manipulating the local dimensionality of spacetime using an elaborate and complex set up of Casimir plates system we could build a nano universe and extract its dark energy concentrated at its boundary. The way to do this economically may be five, ten or more years of experimental work using the modern developments of cutting 
edge nanotechnology [1]. Never the less, the promise of near to infinite, clean, free energy is a goal worth any effort and the financial risks are minimal compared to the possible gains, so let the present modest steps be the first into this new world of a nano, Casimir-dark energy reactor.

The paper is structured as follows: In Section 2 immediately after the introduction we give vital information regarding the theory used, namely E-infinity Cantorian spacetime theory [30]-[40]. In Section 3 the vital idea of a one sided Möbius-like boundary of the holographic boundary of the universe is introduced. Sections 4 and 5 discuss the experimental role of nano technology in converting a mathematical model into a real, useful device to produce clean energy. Section 6 gives our main theoretical results and finally section 7 is the general conclusion.

\section{Background Information}

Based on his E-infinity Cantorian spacetime theory [1]-[21], it was recently argued by the author that the Casimir effect is a local manifestation of the quantum wave while dark energy is the global manifestation of the same [1]. The only difference is that of the details of the boundary conditions [1] [2]. It was further reasoned by the author that the universe as a whole has a one sided boundary akin to that of higher dimensional Möbius band and consequently the "local" Casimir effect ramifies at this one sided boundary located at infinity to produce the negative gravity pressure of the conjectured dark energy [1]-[3]. In other words, three rather mysterious physical notions are tied together and explained in terms of each other. At the top resides the quantum wave [4], which is not a mathematical artifact [4]-[8] but according to E-infinity theory of dark energy, a real physical entity fully described by the empty set fixed by Connes-El Naschie bi dimension $\left(-1, \phi^{2}\right)$ where $\phi=1 /(\sqrt{5}+1)$ [1]-[8]. On the other hand the gradient caused by different wave energy density in different bounded regions of space compared to the unbounded outside of the same space is behind the Casimir forces which in the limit can be show to be equal to the difference between the quantum zero set $(0, \phi)$ and the wave empty set $\left(-1, \phi^{2}\right)$ leading to $\phi-\phi^{2}=\phi^{3}$ topological energy pressure [1] [5]-[8]. Finally at the edge of the universe there is only internal Casimir quantum wave pressure not balanced by outside pressure which is the dark energy concentration of 96 percent as per the consequences of Dvoretzky's theorem and the present author's dissection of Einstein's $E=m c^{2}$ to $E(O)=m c^{2} / 22$ for ordinary energy of the quantum particle and $E(D)=m c^{2}(21 / 22)$ for the dark energy of the quantum wave [7] [8].

The sources of the ideas contained in the present work go back to many years ago when we attempted to improve on the traditional fast and slow fission reactors using the modern mathematics of fractals and nonlinear dynamics [9]-[17]. The second source is our recent reinterpretation of Einstein's $E=m c^{2}$ and finally the third source is the unexpected results of the earlier mentioned Dvoretzky's theorem of Banach spaces [3] [6]. However in the final analysis building an actual reactor could not be possible, not even in principle, without first a sound theory [1]-[56] and second the combination of modern nanotechnology and state of the art Casimir effect experimentation [18]-[24]. In addition a reasonable amount of imaginative thinking similar to that of the man who is famed for inventing the $20^{\text {th }}$ century is also recommended [49] [57].

As we mention at the beginning of our introduction, to keep the present paper short and yet to cover the large amount of the needed prerequisites we opted for a condensed presentation coupled to a large number of references. We recommend to start by reading Ref. 1 and Ref. 60, then it is a personal choice of how to proceed after that.

\section{The Boundary of the Holographic Boundary of the Universe and the Empty Set}

The holographic boundary theory goes back to the pioneering work of 'tHooft and Susskind [25]-[27]. On the other hand the principle that the boundary of a boundary is zero goes back to the out of the box thinking of J. A. Wheeler [28]. Pushing their ideas further still, it became obvious to the present author that the boundary of the holographic boundary is not only a zero limit set but actually a hierarchy of empty and emptier still sets ramifying at a most general form of a one sided higher dimensional Möbius band [28]-[33]. This limit set resembles a fundamental polyhedron group or better still, a Schottky-Kleinian group [29]-[33] which changes the topology of our conventional Casimir experiment to that of a sphere with internal Casimir pressure inflating the balloon-like universe and makes it expand into the surrounding "nothingness" fixed by the well known $E$-infinity 
formula $d_{c}^{(-\infty)}=\phi^{\infty}=0$ where $\phi=1 /(\sqrt{5}+1)$ [34]. From the preceding elementary reasoning it is clear that

Casimir-effect and dark energy have the same cause, namely the topology of a Banach-spacetime like manifold and the only difference is the difference of local exophysics and global endophysics and the respective associated boundary conditions [1] [2]. There is already a vast body of literature on the subject published in the last three years alone by the present author and his associates [1]-[56]. However, what we are aiming at in the present paper is to point out the way to move from theory to useful, practical application of which nothing could be more important and pressing than building a free energy reactor, based on real science rather than wishful thinking. Thus we will combine the dreams of visionaries like N. Tesla with hard nosed modern mathematics and physics which were not yet available in the time of Tesla [49].

\section{Enter Nanotechnology}

There has been no want of imaginative experimental set ups for measuring, testing and visualizing the Casimir effect since it was proposed by Dutch physicist, H. Casimir [22]-[24]. In recent years nanotechnology invaded all scientific fields and played a significant role in Casimir effect experiments. Thanks to E-infinity we now know that the true physical-mathematical connection between dark energy and the Casimir effect. A natural consequence of this discovered reality of the quantum wave, is rendering it a relatively simple task to find a way to harness dark energy or Casimir energy. Of course this "simple" is extremely difficult but no longer impossible.

We can start with a highly complex sub-structuring of space using nano tubes and nano particles and create that way nanosphere packing modelling the moonshine conjecture which relates superstrings to other fields of theoretical physics. We presently have, in embryonic form, the main idea of constructing a nano universe and extracting dark energy from its nano boundary of its holographic boundary. Our program to actually extract energy from such a nano reactor may still need five or more years but the road is marked and reasonably clear. It is only at the edge of the universe that $96 \%$ of the energy resides as dark energy. However we could create many nano universes from which its $96 \%$ energy concentration could be extracted without actually reaching to the boundary of our universe [3]-[8].

\section{Laboratory Work between Real and Gedanken Experiments}

In non-commutative geometry as well as E-infinity theory, the Penrose universe plays a significant role as a generic concrete model for both theories [50]-[53]. On the other hand Penrose universe or Penrose fractal tiling is basically a quasi-crystal mathematical model with the forbidden 5-fold symmetry [53] [54]. This form of matter not found naturally on earth, was produced experimentally by the great Israeli engineer D. Schechtman, who after facing a long period of fierce opposition from high profile scientists, for instance Nobel Laureate Linus Pauling, was rehabilitated and bestowed with a Nobel Prize. The 5 fold symmetry could be thought of theoretically as five Kaluza-Klein dimensions and using nano particles and nano tubes combinations we could build in the lab a nano holographic universe [5]-[8] akin to our own from which energy could be experimented with and extracted. For sure it will be a journey in unchartered seas with many trials and errors but sooner or later we will find out the right road to a Casimir dark energy nano reactor [1] [22]. There are other conceivable ways of producing artificial nano universes with high dimensionality for Dvoretzky's theorem to be applicable. For instance we could use Ji-Huan He's ten dimensional polytope [42] as a skeleton to grow on it a hierarchy of nano particles using the methods applied in the clustering of diffusion limited aggregation. In other words, we can let our scientific imagination run free but checked with $E$-infinity mathematical rigor and nanotechnological facts.

\section{The Topological Casimir-Dark Energy Density Using E-Infinity Theory}

It may come as a pleasant mild surprize that exact limits could easily be established for Casimir-dark energy using nothing more than the topology of our E-infinity Cantorian spacetime [56] [60]. We can do this in a variety of ways which are essentially tautologies leading to the same basic conclusion in the limit. Thus we could view the energy density of the space outside the two Casimir plates as that of Einstein's $E=m c^{2}$ density, i.e. $\gamma($ Einstein $)=1$. Inside the plate the energy density in the limit could only be a statical, quasi potential energy of the quantum particle, i.e. $E=m c^{2} / 22$ and consequently $\gamma(0)=1 / 22$. It follows then that the net pressure of 
the Casimir plates must be $1-(1 / 22)=21 / 22$ which is, in the meantime rather well known, as the dark energy density of spacetime. A second way to interpret the same situation and reach the same result is to argue that within the Casimir plates there is no "space" except for the empty set with a Hausdorff dimension $\phi^{2}$ where $\phi=1 /(\sqrt{5}+1)$. Outside on the other hand we have the zero set. The difference is a net $\phi-\phi^{2}=\phi^{3}$ which is the universal fluctuation of spacetime and simply the reciprocal value of its Hausdorff dimension $\left(1 / \phi^{3}\right)=4+\phi^{3}$ [3] [52] [56]. Finally we could see the situation as the difference of the completely empty set in the limit, i.e. zero between the Casimir plates and the spacetime fluctuation $\phi^{3}$ [60]. That way the Casimir effect could be set in the limit equal to $\phi^{3}$ and may easily be seen to be a relative to the Immirzi parameter $\phi^{6}$ and the Unruh temperature $\phi^{4}$ apart of Hardy's entanglement $\phi^{5}$, i.e. a member of a generalized quantumtopological entanglement family [60].

\section{Discussion, Conclusion and a Plea for a Peaceful Future}

It would be a gross error to place the present nano reactor proposal within the context of science fiction. There is definitely a trivial element of speculation and trial and error but that is all. Exploding stars and galaxies are scientific facts. Consequently to presume that these are only topological defects in to near infinitely large spacetime is not outlandish nor science fiction [54]-[56]. In fact the near identity of the Casimir effect and dark energy and the fact that both originate from the quantum wave aspect of quantum mechanics clearly shows to any open minded scientific thinker that to pursue clean free energy is a scientific real and reachable aim. The $4.5 \%$ of ordinary energy in the universe is nothing but the multiplicative volume of a five dimensional K-K zero set while the 95.5\% dark energy is the additive volume of the same 5D Kaluza-Klein empty set [34]. Seen that way we think that making humanity free from oil and traditional sources of energy is a higher and moral aim worth investing heavily in for what is a million or even billion dollar research grant funding compared to the three trillion dollar Iraq war [59]. In fact the highly enlightened rules of the United Arab Emirates are already looking towards a future free of oil based energy [61]. It was Nobel Laureate in Economics, Prof. J. Stiglitz who calculated with Prof. L. Bilmes the true cost of the Iraq war for the USA. The staggering three trillion dollars do not actually include the loss and destruction for the economy of the entire world. The author dares to say with a tongue in cheek, that the mere sight of only one trillion dollars funding for our nano Casimir-dark energy reactor is sufficient to make this reactor spontaneously pop out of spacetime like virtual particles!

The author, who was born and raised in the Middle East with its unrivalled rich history and unparalleled chequered present day politics feels morally obliged to call all the governments of the region to participate in a new dawn of science and life.

\section{References}

[1] El Naschie, M.S. (2015) Three Quantum Particles Hardy Entanglement from the Topology of Cantorian-Fractal Spacetime and the Casimir Effect as Dark Energy_A Great Opportunity for Nanotechnology. American Journal of Nano Research and Applications, 3, 1-5.

[2] El Naschie, M.S. (2014) Casimir-Like Energy as a Double Eigenvalue of Quantumly Entangled System Leading to the Missing Dark Energy Density of the Cosmos. International Journal of High Energy Physics, 1, 55-63.

[3] El Naschie, M.S. (2014) The Measure Concentration of Convex Geometry in a Quasi Banach Spacetime behind the Supposedly Missing Dark Energy of the Cosmos. American Journal of Astronomy \& Astrophysics, 2, 72-77.

[4] Slezak, M. (2015) Quantum Wave Function Gets Real. New Scientist, 7 February, 14. http://dx.doi.org/10.1016/S0262-4079(15)60242-1

[5] El Naschie, M.S. (2015) Dark Energy and Its Cosmic Density from Einstein's Relativity and Gauge Fields Renormalization Leading to the Possibility of a New 'tHooft Quasi Particle. The Open Astronomy Journal, 8, 1-17. http://dx.doi.org/10.2174/1874381101508010001

[6] El Naschie, M.S. (2015) Banach Spacetime-Like Dvoretzky Volume Concentration as Cosmic Holographic Dark Energy. International Journal of High Energy Physics, 2, 13-21.

[7] El Naschie, M.S. (2014) From $E=m c^{2}$ to $E=m c^{2} / 22-$ A Short Account of the Most Famous Equation in Physics and Its Hidden Quantum Entanglement Origin. Journal of Quantum Information Science, 4, 284-291. http://dx.doi.org/10.4236/jqis.2014.44023

[8] El Naschie, M.S. (2014) The Hidden Quantum Entanglement Roots of $E=m c^{2}$ and Its Genesis to $E=m c^{2} / 22$ plus 
$m c^{2}(21 / 22)$ Confirming Einstein's Mass-Energy Formula. American Journal of Electromagnetics and Applications, 2, 39-44. http://dx.doi.org/10.11648/j.ajea.20140205.11

[9] El Naschie, M.S. (1999) From Implosion to Fractal Spheres. A Brief Account of the Historical Development of Scientific Ideas Leading to the Trinity Test and Beyond. Chaos, Solitons \& Fractals, 10, 1955-1965. http://dx.doi.org/10.1016/S0960-0779(99)00030-2

[10] El Naschie, M.S. and Al Athel, S. (2000) Estimating the Eigenvalue of Fast Reactors and Cantorian Space. Chaos, Solitons \& Fractals, 11, 1957-1961. http://dx.doi.org/10.1016/S0960-0779(99)00069-7

[11] El Naschie, M.S. (2000) On Nishina’s Estimate of the Critical Mass for Fission and Early Nuclear Research in Japan. Chaos, Solitons \& Fractals, 11, 1809-1818. http://dx.doi.org/10.1016/S0960-0779(99)00172-1

[12] El Naschie, M.S. (2000) Remarks on Heisenberg's Farm-Hall Lecture on the Critical Mass of Fast Neutron Fission. Chaos, Solitons \& Fractals, 11, 1327-1333. http://dx.doi.org/10.1016/S0960-0779(99)00136-8

[13] El Naschie, M.S. and Hussein, A. (2000) On the Eigenvalue of Nuclear Reaction and Self-Weight Buckling. Chaos, Solitons \& Fractals, 11, 815-818. http://dx.doi.org/10.1016/S0960-0779(99)00106-X

[14] El Naschie, M.S. (2000) Elastic Buckling Loads and Fission Critical Mass as an Eigenvalue of a Symmetry Breaking Bifurcation. Chaos, Solitons \& Fractals, 11, 631-629. http://dx.doi.org/10.1016/S0960-0779(99)00063-6

[15] El Naschie, M.S. (2000) On the Zel'dovich-Khuriton Critical Mass for Fast Fission. Chaos, Solitons \& Fractals, 11, 819-824. http://dx.doi.org/10.1016/S0960-0779(99)00113-7

[16] El Naschie, M.S. (2000) On the Eigenvalue of Transport Reaction Involving Fast Neutrons. Chaos, Solitons \& Fractals, 11, 929-934. http://dx.doi.org/10.1016/S0960-0779(99)00066-1

[17] El Naschie, M.S. (2000) Heisenberg’s Critical Mass Calculations for an Explosive Nuclear Reaction. Chaos, Solitons \& Fractals, 11, 987-997. http://dx.doi.org/10.1016/S0960-0779(99)00110-1

[18] El Naschie, M.S. (1998) Chaos and Fractals in Nano and Quantum Technology. Chaos, Solitons \& Fractals, 9, 17931802.

[19] El Naschie, M.S. (2006) Nanotechnology for the Developing World. Chaos, Solitons \& Fractals, 30, 769-773. http://dx.doi.org/10.1016/j.chaos.2006.04.037

[20] El Naschie, M.S. (2007) The Political Economy of Nanotechnology and the Developing World. International Journal of Electrospun Nanofibers and Applications, 1, 41-50.

[21] El Naschie, M.S. (1998) Some Tentative Proposals for the Experimental Verification of Cantorian Micro Spacetime. Chaos, Solitons \& Fractals, 9, 143-144. http://dx.doi.org/10.1016/S0960-0779(97)00175-6

[22] Johnston, H. (2012) Physicists Solve Casimir Conundrum. Physicsworld.com, July 18, 2012.

[23] Rencroft, S. and Swain, J. (1998) What Is the Casimir Effect? Scientific American, June 22, 1998.

[24] Wongjun, P. (2015) Casimir Dark Energy, Stabilization and the Extra Dimensions and Gauss-Bonnet Term. The European Physical Journal C, 75, 6.

[25] El Naschie, M.S. (2007) A Review of Application and Results of E-Infinity. International Journal of Nonlinear Science \& Numerical Simulation, 8, 11-20. http://dx.doi.org/10.1515/IJNSNS.2007.8.1.11

[26] Smolin, L. (2001) The Strong and the Weak Holographic Principles. Nuclear Physics B, 601, 209-247. http://dx.doi.org/10.1016/S0550-3213(01)00049-9

[27] El Naschie, M.S. (2006) Holographic Dimensional Reduction: Centre Manifold Theorem and E-Infinity. Chaos, Solitons \& Fractals, 29, 816-822. http://dx.doi.org/10.1016/j.chaos.2006.01.013

[28] Misner, C., Thorne, K. and Wheeler, J.A. (1973) Gravitation. Freeman, New York.

[29] El Naschie, M.S. (2003) Kleinian Groups in E-Infinity and Their Connection to Particle Physics and Cosmology. Chaos, Solitons \& Fractals, 16, 637-649. http://dx.doi.org/10.1016/S0960-0779(02)00489-7

[30] El Naschie, M.S. (2005) A Guide to the Mathematics of E-Infinity Cantorian Spacetime Theory. Chaos, Solitons \& Fractals, 25, 955-964. http://dx.doi.org/10.1016/j.chaos.2004.12.033

[31] El Naschie, M.S. (2004) The Concepts of E-Infinity: An Elementary Introduction to the Cantorian-Fractal Theory of Quantum Physics. Chaos, Solitons \& Fractals, 22, 495-511. http://dx.doi.org/10.1016/j.chaos.2004.02.028

[32] El Naschie, M.S. (2003) Complex Vacuum Fluctuation as a Chaotic “Limit” Set of Any Kleinian Group Transformation and the Mass Spectrum of High Energy Particle Physics via Spontaneous Self Organization. Chaos, Solitons \& Fractals, 17, 631-638. http://dx.doi.org/10.1016/S0960-0779(02)00630-6

[33] El Naschie, M.S. (2003) Modular Groups in Cantorian E-Infinity High Energy Physics. Chaos, Solitons \& Fractals, 16, 353-366. http://dx.doi.org/10.1016/S0960-0779(02)00440-X

[34] El Naschie, M.S. (1994) On Certain “Empty” Cantor Sets and Their Dimensions. Chaos, Solitons \& Fractals, 4, 293- 
296. http://dx.doi.org/10.1016/0960-0779(94)90152-X

[35] He, J.-H., Xu, L., Zhang, L.-N. and Wu, X.-H. (2007) Twenty Six Dimensional Polytope and High Energy Spacetime Physics. Chaos, Solitons \& Fractals, 33, 5-13. http://dx.doi.org/10.1016/j.chaos.2006.10.048

[36] El Naschie, M.S. (1994) Is Quantum Space a Random Cantor Set with a Golden Mean Dimension at the Core? Chaos, Solitons \& Fractals, 4, 177-179. http://dx.doi.org/10.1016/0960-0779(94)90141-4

[37] El Naschie, M.S. (2008) Mathematical Foundation of E-Infinity via Coxeter and Reflection Groups. Chaos, Solitons \& Fractals, 37, 1267-1268. http://dx.doi.org/10.1016/j.chaos.2008.02.001

[38] El Naschie, M.S. (1995) Banach-Tarski Theorem and Cantorian Micro Spacetime. Chaos, Solitons \& Fractals, 5, 1503-1508. http://dx.doi.org/10.1016/0960-0779(95)00052-6

[39] El Naschie, M.S. (1995) On the Initial Singularity and the Banach-Tarski Theorem. Chaos, Solitons \& Fractals, 5, 1391-1392. http://dx.doi.org/10.1016/0960-0779(95)99645-2

[40] El Naschie, M.S. (1998) COBE Satellite Measurement, Hyper Spheres, Superstrings and the Dimension of Spacetime. Chaos, Solitons \& Fractals, 9, 1445-1471. http://dx.doi.org/10.1016/S0960-0779(98)00120-9

[41] El Naschie, M.S. (2001) Infinite Dimensional Branes and the E-Infinity Topology of Heterotic Superstrings. Chaos, Solitons \& Fractals, 12, 1047-1055. http://dx.doi.org/10.1016/S0960-0779(00)00130-2

[42] El Naschie, M.S. (2007) Ji-Huan He’s Ten Dimensional Polytope and High Energy Particle Physics. International Journal of Nonlinear Science \& Numerical Simulation, 8, 475-476. http://dx.doi.org/10.1515/IJNSNS.2007.8.4.475

[43] El Naschie, M.S. (1999) Hyper-Dimensional Geometry and the Nature of Physical Spacetime. Chaos, Solitons \& Fractals, 10, 155-158. http://dx.doi.org/10.1016/S0960-0779(98)00235-5

[44] Finkelstein, D. (1982) Quantum Sets and Clifford Algebras. International Journal of Theoretical Physics, 21, $489-503$.

[45] El Naschie, M.S. (2002) Derivation of the Threshold and Absolute Temperature $T_{c}=273.16 \mathrm{k}$ from the Topology of Quantum Spacetime. Chaos, Solitons \& Fractals, 14, 1117-1120. http://dx.doi.org/10.1016/S0960-0779(02)00053-X

[46] El Naschie, M.S. (2008) Quarks Confinement via Kaluza-Klein Theory as a Topological Property of Quantum-Classical Spacetime Phase Transition. Chaos, Solitons \& Fractals, 35, 825-829. http://dx.doi.org/10.1016/j.chaos.2007.08.057

[47] El Naschie, M.S. (2002) On a Class of General Theories for Higher Energy Particle Physics. Chaos, Solitons \& Fractals, 14, 649-668. http://dx.doi.org/10.1016/S0960-0779(02)00033-4

[48] He, J.-H. (2009) Hilbert Cube Model for Fractal Spacetime. Chaos, Solitons \& Fractals, 42, 2754-2759. http://dx.doi.org/10.1016/j.chaos.2009.03.182

[49] Lomas, R. (1999) The Man Who Invented The Twentieth Century, Nicola Tesla, Forgotten Genius of Electricity. Headline Books, London.

[50] Helal, M., Marek-Crnjac, L. and He, J.-H. (2013) The Three Page Guide to the Most Important Results of M.S. El Naschie’s Research in E-Infinity Quantum Physics. Open Journal of Microphysics, 3, 141-145. http://dx.doi.org/10.4236/ojm.2013.34020

[51] El Naschie, M.S. (2004) A Review of E-Infinity Theory and the Mass Spectrum of High Energy Physics. Chaos, Solitons \& Fractals, 19, 209-236. http://dx.doi.org/10.1016/S0960-0779(03)00278-9

[52] El Naschie, M.S. (2009) The Theory of Cantorian Spacetime and High Energy Particle Physics (An Informal Review). Chaos, Solitons \& Fractals, 41, 2635-2646. http://dx.doi.org/10.1016/j.chaos.2008.09.059

[53] Marek-Crnjac, L. and He, J.-H. (2013) An Invitation to El Naschie’s Theory of Cantorian Spacetime and Dark Energy. International Journal of Astronomy and Astrophysics, 3, 464-471.

[54] El Naschie, M.S. (2013) A Resolution of Cosmic Dark Energy via Quantum Entanglement Relativity Theory. Journal of Quantum Information Science, 3, 23-26. http://dx.doi.org/10.4236/jqis.2013.31006

[55] El Naschie, M.S. (2013) What Is the Missing Dark Energy in a Nutshell and the Hawking-Hartle Quantum Wave Collapse. International Journal of Astronomy \& Astrophysics, 3, 205-211. http://dx.doi.org/10.4236/ijaa.2013.33024

[56] El Naschie, M.S. (2013) Topological-Geometrical and Physical Interpretation of the Dark Energy of the Cosmos as a “Halo” Energy of the Schrodinger Quantum Wave. Journal of Modern Physics, 4, 591-596. http://dx.doi.org/10.4236/jmp.2013.45084

[57] Peat, F.D. (1983) In Search of Nikola Tesla. Ashgrove Publications, London \& Bath.

[58] Susskind, L. and Lindesay, J. (2005) The Holographic Universe. World Scientific, Singapore.

[59] Stiglitz, J. and Bilmes, L. (2008) The Three Trillion Dollar War: The True Cost of the Iraq Conflict. Allen-Lane, Penguin Books, London.

[60] El Naschie, M.S. (2013) A Unified Newtonian-Relativistic Quantum Resolution of Supposedly Missing Dark Energy 
of the Cosmos and the Constancy of the Speed of Light. International Journal of Modern Nonlinear Theory \& Application, 2, 43-54. http://dx.doi.org/10.4236/ijmnta.2013.21005

[61] Malek, C. (2015) Abu Dhabi Crown Prince Details UAE Leaders’ Vision of Future without Oil. The National Newspaper, UAE, February 10th, 2015.

http://www.thenational.ae/uae/government/abu-dhabi-crown-prince-details-uae-leaders-vision-of-future-without-oil?ut m_content='\%20vision\%20of\%20future\%20without\%20oil

[62] Zee, A. (2003) Quantum Field Theory in a Nutshell. Princeton University Press, Princeton.

[63] Duplantier, B. and Rivasseau, V., Eds. (2003) Vacuum Energy-Renormalization. Birkhauser, Basel.

[64] Milonni, P.W. (1994) The Quantum Vacuum. Academic Press, Boston.

[65] Parsegian, V.A. (2006) Van der Waals Forces. Cambridge University Press, Cambridge.

[66] Huang, K. (2007) Fundamental Forces of Nature. World Scientific, Singapore.

[67] Wapner, L.M. (2005) The Pea and the Sun. A.K. Peters Ltd., Wellesley.

[68] El Naschie, M.S. (1995) Banach-Tarski Theorem and Cantorian Micro Spacetime. Chaos, Solitons \& Fractals, 5, 15031508. http://dx.doi.org/10.1016/0960-0779(95)00052-6 